\title{
Fast Multipole Method for Scattering from an Arbitrary PEC Target Above or Buried in a Lossy Half Space
}

Norbert Geng, Member, IEEE, Anders Sullivan, Member, IEEE, and Lawrence Carin, Fellow, IEEE

\begin{abstract}
The fast multipole method (FMM) was originally developed for perfect electric conductors (PECs) in free space, through exploitation of spectral properties of the free-space Green's function. In the work reported here, the FMM is modified, for scattering from an arbitrary three-dimensional (3-D) PEC target above or buried in a lossy half space. The "near" terms in the FMM are handled via the original method-of-moments (MoM) analysis, wherein the half-space Green's function is evaluated efficiently and rigorously through application of the method of complex images. The "far" FMM interactions, which employ a clustering of expansion and testing functions, utilize an approximation to the Green's function dyadic via real image sources and far-field reflection dyadics. The half-space FMM algorithm is validated through comparison with results computed via a rigorous MoM analysis. Further, a detailed comparison is performed on the memory and computational requirements of the MoM and FMM algorithms for a target in the vicinity of a half-space interface.
\end{abstract}

Index Terms-Method of moments, scattering.

\section{INTRODUCTION}

$\mathbf{R}$ ESEARCHERS have directed significant attention toward development of numerical scattering models, with the method of moments (MoM) representing a prominent example [1]-[7]. For an MoM problem with $N$ unknowns, the memory requirements are of order $O\left(N^{2}\right)$, while the computational complexity depends on whether a direct (LU-decomposition) or iterative (conjugate gradient [2], [8]) matrix solver is applied, the former requiring $O\left(N^{3}\right)$ and the latter $P \cdot O\left(N^{2}\right)$ computations, with $P$ the number of iterations required to achieve convergence. To reduce computational complexity, several researchers have undertaken the development of modified integral-equation solvers [9]-[17], in an effort to achieve better computational efficiency and reduced memory requirements. For example, the adaptive integral method (AIM) [12] exploits properties of the FFT, and researchers have demonstrated memory requirements and computational complexity of $O\left(N^{1.5}\right)$ and $O\left(N^{1.5} \log N\right)$, respectively [12]. Further, there has been significant interest in the fast multipole method (FMM). The simplest two-level (single-stage) FMM [13], [14] has $O\left(N^{1.5}\right)$ computational complexity and memory

Manuscript received January 5, 1999; revised September 27, 2000.

N. Geng is with the University of Karlsruhe, Institute for Microwaves and Electronics, D-76128 Karlsruhe, Germany.

A. Sullivan and L. Carin are with the Duke University, Department of Electrical and Computer Engineering, Durham, NC 27708-0291 USA.

Publisher Item Identifier S 0018-926X(01)03184-2. requirements, while a multilevel extension [15]-[17] reduces these numbers to $O(N \log N)$.

While the AIM and FMM algorithms represent promising tools, they have heretofore been applied primarily to free-space scattering [12], [13]-[17], two-dimensional (2-D) analysis in layered media [18], or quasi-planar three-dimensional (3-D) problems in circuit and antenna design [19]. There are many applications for which the free-space model or a quasi-planar approximation are inappropriate. For example, there has been significant interest in radar sensing of buried targets, such as mines [20], unexploded ordnance (UXO) [21], drums [22], etc. In this paper we concentrate on the two-level FMM, with the goal of extending it to the case of a target in the vicinity of a lossy half space.

The FMM clusters the MoM basis elements into $M$ groups [13], [14], and the interactions between distant groups ("far" interactions) are handled by exploiting features of the FMM spectral propagator [13], [14], discussed further below. "Near" interactions are handled in a manner analogous to a conventional MoM analysis [3], [5], [7]. For the calculation of these "near" interactions, we rigorously evaluate the dyadic half-space Green's function [6], [25] via the complex-image technique [26]-[29].

We have developed an approximate but highly accurate method for evaluating the "far" FMM interactions within the context of a half-space problem. In particular, if the target is entirely above or below the half space, the MoM and FMM analyses only require the Green's function for source and observer in the same region. Under this circumstance, each component of the dyadic Green's function can in general be represented in terms of a direct and reflected term [30], [31]. The direct term is handled as in the conventional free-space FMM [13], [14]. Further, the reflected term corresponds to radiation from the expansion function, reflection at the half-space interface, and subsequent propagation to the testing function. If the expansion and testing functions are relatively distant, than this term can be evaluated approximately asymptotically [30], [31]. The reflected term can therefore be represented in terms of an image in real space, with an appropriate amplitude, dictated by the polarization-dependent reflection coefficient.

\section{THEORY}

\section{A. Integral Equation and MoM Formulation}

We utilize an electric-field integral equation (EFIE) formulation [2], [3], [5], which restricts its utility somewhat, due to 

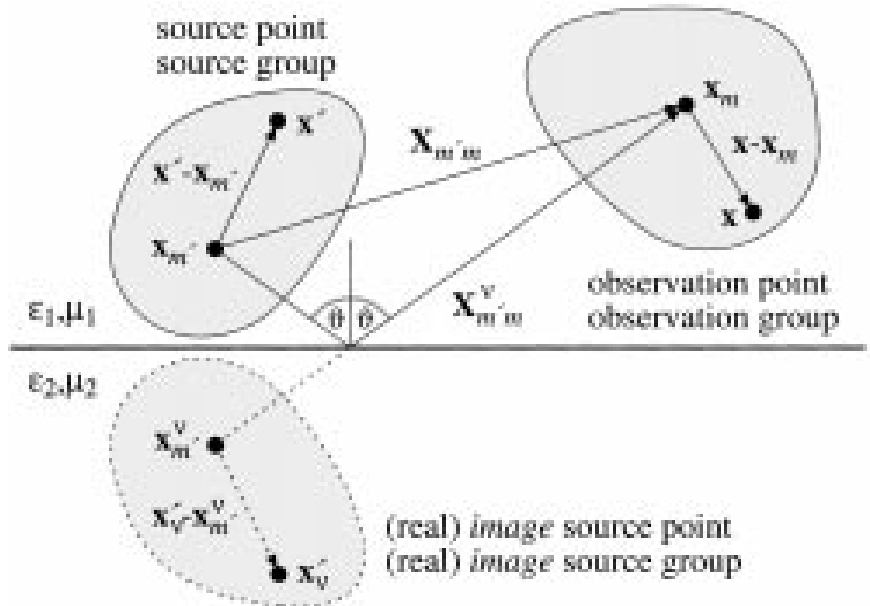

Fig. 1. Geometry for source and observation group in 3-D FMM and generalization to a half-space environment using real images to account for "far" interface interactions.

spurious internal resonances (for closed targets) [2]. However, it has been demonstrated previously [23] that when a closed target is embedded in a lossy medium (e.g., soil), these internal resonances are damped significantly and they therefore present less of a problem computationally. We note, however, that the general FMM formulation can also be applied to a combined field integral equation (CFIE) [2], [15], which is not beset by internal-resonance problems.

The half-space EFIE for a perfectly conducting 3-D target situated entirely in layer $\mathrm{i}=1$ (e.g., air) or layer $i=2$ (e.g., soil) of a half-space environment (Fig. 1) can be expressed as [6]

$$
\begin{aligned}
\hat{\boldsymbol{n}} \times \boldsymbol{E}^{\mathrm{inc}}(\boldsymbol{x})=\hat{\boldsymbol{n}} \times j \omega \mu_{i} \iint_{S^{\prime}}\left[\boldsymbol{I}+\frac{\nabla \nabla}{k_{i}^{2}}\right] \\
\cdot \boldsymbol{G}_{\mathrm{Aii}}\left(\boldsymbol{x}, \boldsymbol{x}^{\prime}\right) \cdot \boldsymbol{J}\left(\boldsymbol{x}^{\prime}\right) d S^{\prime}
\end{aligned}
$$

or, alternatively, in the mixed potential form of the electric field integral equation (MPIE) [6]

$$
\begin{aligned}
\hat{\boldsymbol{n}} \times \boldsymbol{E}^{i n c}(\boldsymbol{x})=\hat{\boldsymbol{n}} \times & {\left[j \omega \mu_{i} \iint_{S^{\prime}} \boldsymbol{K}_{A i i}\left(\boldsymbol{x}, \boldsymbol{x}^{\prime}\right) \cdot \boldsymbol{J}\left(\boldsymbol{x}^{\prime}\right) d S^{\prime}\right.} \\
& \left.-\frac{\nabla}{j \omega \varepsilon_{i}} \iint_{S^{\prime}} K_{\phi e}^{i i}\left(\boldsymbol{x}, \boldsymbol{x}^{\prime}\right) \nabla^{\prime} \cdot \boldsymbol{J}\left(\boldsymbol{x}^{\prime}\right) d S^{\prime}\right]
\end{aligned}
$$

where $\boldsymbol{x}$ is on and $\hat{\boldsymbol{n}}$ is a unit vector normal to the target surface; $\epsilon_{i}=\epsilon_{i}^{\prime}-j \sigma / \omega, \mu_{i}$ and $k_{i}$ represent (in general complex) permittivity, permeability, and wavenumber of the medium in which the target resides (region $i$ ); $\omega$ is the angular frequency $(\exp (j \omega t)$ time dependence is assumed and suppressed); and $I$ represents the unit dyadic. Details on the dyadic half-space Green's functions $\boldsymbol{G}_{A i i}$ and $\boldsymbol{K}_{A i i}$, and on the scalar Green's function $K_{\phi e}^{i i}$, have been given by Michalski and Zheng [6], where we use their formulation C. The EFIE in (1) is valid for a general layered medium [6], but here we only consider a half-space, for simplicity (see also explanation in Section II-C).
Like in a conventional MoM solution, the unknown surface current $J$ is expanded by a set of basis (or expansion) functions $\boldsymbol{b}_{n^{\prime}}\left(\boldsymbol{x}^{\prime}\right)$, where we use the well-known RWG vector basis functions introduced in [3], defined on adjacent triangles representing the target's surface. Testing the EFIE (1) with a set of weighting (or testing) functions $w_{n}(\boldsymbol{x})$ tangential to the scatterer surface results in $N$ linear equations $[Z] \cdot I=V$ for the unknown current coefficients $I_{n^{\prime}}$, where the $N \times 1$ driving vector $V$ represents the tangential electric field of the incident wave, tested on the target surface. The elements of the $N \times N$ impedance matrix $[Z]$ are given by

$$
\begin{gathered}
Z_{n n^{\prime}}=j \omega \mu_{i} \iint_{S} \iint_{S^{\prime}} w_{n}(\boldsymbol{x}) \cdot\left[\boldsymbol{I}+\frac{\nabla \nabla}{k_{i}^{2}}\right] \\
\cdot G_{A i i}\left(\boldsymbol{x}, \boldsymbol{x}^{\prime}\right) \cdot \boldsymbol{b}_{n^{\prime}}\left(\boldsymbol{x}^{\prime}\right) d S^{\prime} d S
\end{gathered}
$$

or

$$
\begin{aligned}
Z_{n n^{\prime}}= & j \omega \mu_{i} \iint_{S} w_{n}(\boldsymbol{x}) \cdot \iint_{S^{\prime}} \mathbf{K}_{\mathbf{A i i}}\left(\boldsymbol{x}, \boldsymbol{x}^{\prime}\right) \cdot \boldsymbol{b}_{\mathbf{n}^{\prime}}\left(\boldsymbol{x}^{\prime}\right) \mathbf{d} \mathbf{S}^{\prime} \mathbf{d} \mathbf{S} \\
& -\frac{1}{j \omega \varepsilon_{i}} \iint_{S} w_{n}(\boldsymbol{x}) \cdot \nabla \iint_{S^{\prime}} \mathbf{K}_{\phi \mathbf{e}}^{\mathbf{i i}}\left(\boldsymbol{x}, \boldsymbol{x}^{\prime}\right) \nabla^{\prime} \\
& \cdot \boldsymbol{b}_{n^{\prime}}\left(\boldsymbol{x}^{\prime}\right) d S^{\prime} d S
\end{aligned}
$$

Using the RWG triangular basis [3] and Galerkin testing, the last term in (2b) can be simplified using integration by parts [3].

In the FMM analysis [13], [14], we divide the computation of (expansion function)-(testing function) interactions into "near" and "far" terms. The "far" interactions are best handled via the form in (1a) and (2a), respectively, while for "near" interactions (MoM part of the FMM [13], [14]) (1b) and (2b) are utilized, rigorously accounting for the dyadic half-space Green's function [6]. The half-space Green's function is evaluated using the method of complex images [26]-[29], thereby avoiding direct numerical evaluation of Sommerfeld integrals [25]. Impedance matrix elements representing these "near" interactions are stored in a sparse matrix denoted [ $\left.Z^{\text {near }}\right]$. The method of complex images for calculating the space-domain Green's function, as well as explicit equations for the impedance matrix elements, including the extraction of self-term singularities, can be found in the literature [26]-[29], [3]-[5].

"Far" interactions, applicable to the FMM framework [13], [14], are best described using (2a) and realizing that [6], [30]

$$
\begin{aligned}
\boldsymbol{G}_{A i i}\left(\boldsymbol{x}, \boldsymbol{x}^{\prime}\right) & =\boldsymbol{I} g_{i}\left(\boldsymbol{x}, \boldsymbol{x}^{\prime}\right)+\Delta G_{A i i}\left(\boldsymbol{x}, \boldsymbol{x}^{\prime}\right) \\
& =\boldsymbol{I} \frac{e^{-j k_{i}\left|\boldsymbol{x}-\boldsymbol{x}^{\prime}\right|}}{4 \pi\left|\boldsymbol{x}-\boldsymbol{x}^{\prime}\right|}+\Delta \boldsymbol{G}_{A i i}\left(\boldsymbol{x}, \boldsymbol{x}^{\prime}\right)
\end{aligned}
$$

where the new dyadic $\Delta G_{A i i}$ is defined as the difference between $G_{A i i}$ and $I g_{i}$ (i.e., $\Delta$ is not an operator). The dyadic $G_{A i i}$ is therefore split into a part which accounts for direct interactions (as in the free-space case, but using a homogeneous background medium with in general complex wave number $k_{i}$ ) and a dyadic accounting for interactions with the interface. Consequently, the matrix elements in (2a) for "far" interactions are also split as $Z_{n n^{\prime}}^{\mathrm{far}}=Z_{n n^{\prime}}^{\text {far, hom }}+\Delta Z_{n n^{\prime}}^{\mathrm{far}}$ with $Z_{n n^{\prime}}=Z_{n n^{\prime}}^{\text {near }}+$ $Z_{n n^{\prime}}^{\text {far }}$. As discussed above, $Z_{n n^{\prime}}^{\text {far }}$ is computed using the form 
in (2a), with $Z_{n n^{\prime}}^{\text {far,hom }}$ employing the homogeneous-medium Green's function $g_{i}$ and $\Delta Z_{n n^{\prime}}^{\mathrm{far}}$ employing $\Delta \boldsymbol{G}_{A i i}$ [see (3)].

\section{B. Free-Space Fast Multipole Method}

As is well known from [13], [14], in the 3-D free-space FMM the scalar Green's function $g_{i}\left(\boldsymbol{x}, \boldsymbol{x}^{\prime}\right)$ is expanded using the addition theorem [32] resulting in the plane wave decomposition

$$
\begin{aligned}
& g_{i}\left(\boldsymbol{x}, \boldsymbol{x}^{\prime}\right) \\
& =\frac{-j k_{i}}{(4 \pi)^{2}} \iint_{4 \pi} e^{-j k_{i} \hat{\boldsymbol{k}} \cdot\left(\boldsymbol{x}-\boldsymbol{x}_{m}\right)} T_{L} \\
& \quad \cdot\left(k_{i} X_{m^{\prime} m}, \hat{\boldsymbol{k}} \cdot \hat{\boldsymbol{X}}_{m^{\prime} m}\right) e^{+j k_{i} \hat{\boldsymbol{k}} \cdot\left(\boldsymbol{x}^{\prime}-\boldsymbol{x}_{m^{\prime}}\right)} d^{2} \hat{\boldsymbol{k}} \\
& T_{L}\left(k_{i} X_{m^{\prime} m}, \hat{\boldsymbol{k}} \cdot \boldsymbol{X}_{m^{\prime} m}\right) \\
& =\sum_{l=0}^{L}(-j)^{l}(2 l+1) h_{l}^{(2)}\left(k_{i} X_{m^{\prime} m}\right) P_{l}\left(\hat{\boldsymbol{k}} \cdot \boldsymbol{X}_{m^{\prime} m}\right)
\end{aligned}
$$

where the distance vector $\boldsymbol{R}=\left|\boldsymbol{x}-\boldsymbol{x}^{\prime}\right|$ has been subdivided into a vector from the source point $\boldsymbol{x}^{\prime}$ to the center $\boldsymbol{x}_{m^{\prime}}$ of a corresponding source group, a vector from an observation group center $\boldsymbol{x}_{m}$ to the observation point $\boldsymbol{x}$, and a vector $\boldsymbol{X}_{m^{\prime} m}$ connecting the group centers ( Fig. 1), i.e., $\boldsymbol{R}=\boldsymbol{x}-\boldsymbol{x}^{\prime}=\left(\boldsymbol{x}-\boldsymbol{x}_{m}\right)+$ $\left(\boldsymbol{x}_{m}-\boldsymbol{x}_{m^{\prime}}-\left(\boldsymbol{x}^{\prime}-\boldsymbol{x}_{m}\right)=\left(\boldsymbol{x}-\boldsymbol{x}_{m}\right)+\boldsymbol{X}_{m^{\prime} m}-\left(\boldsymbol{x}^{\prime}-\boldsymbol{x}_{m^{\prime}}\right)\right.$. Therefore, the target surface is first partitioned into groups $m=$ $1, \cdots, M$, each of which has an average number of $A_{m} \approx N / M$ basis/weighting functions. Inside group $m$ the elements are labeled as $\alpha=1, \cdots, A_{m}$ [13], [14]. The group information $n(m)$, is stored in matrix form. As was shown in [13] and [14], for the two-level FMM described here, the optimal number of groups (to minimize CPU and RAM) is given by $M \propto \sqrt{N}$. Approximate equations for the number of terms $L$ needed in (4b) are given in [13], [14], [17] for the case of a lossless environment with $k_{i}=k_{0}$ (vacuum). For a lossy background medium (e.g., soil) more terms are required, as discussed further in Section II-C. Therefore, in our implementation $L$ is determined adaptively, depending on the FMM grouping scheme and the (complex) wavenumber. For the accurate numerical integration over the solid angle $4 \pi$ in (4a), a quadrature rule with $K=2 L^{2}$ points is applied [13], [14]; although more efficient quadrature rules are available [33].

\section{Extension to Half-Space Fast Multipole Method}

In addition to the evaluation of the dyadic Green's function in the calculation of $\left[\mathbf{Z}^{\text {near }}\right]$ (here using the method of complex images [26]-[29]), it is essential to include the effects of the "far" interface interactions represented by $\left[\Delta Z^{\text {far }}\right]$ [see (3)]. Using complex images, each term $\Delta G_{A i i}^{x x}, \Delta G_{A i i}^{z z}$, and $Q_{A i i}$ in the space-domain half-space dyadic Green's function [6]

$$
\begin{aligned}
\Delta G_{A i i}\left(\boldsymbol{x}, \boldsymbol{x}^{\prime}\right)= & (\hat{\boldsymbol{x}} \hat{\boldsymbol{x}}+\hat{\boldsymbol{y}} \hat{\boldsymbol{y}}) \Delta G_{A i i}^{x x}+\left(\hat{z} \hat{\boldsymbol{x}} \frac{\partial}{\partial x}+\hat{z} \hat{\boldsymbol{y}} \frac{\partial}{\partial y}\right) Q_{A i i} \\
& +\hat{z} \hat{z} \Delta G_{A i i}^{z z}
\end{aligned}
$$

can be expressed in terms of a sum of free-space Green's functions [26]-[29] with image sources located in complex space.

The plane wave expansion (4) used in the 3-D free-space FMM remains valid for this more general case of a complex

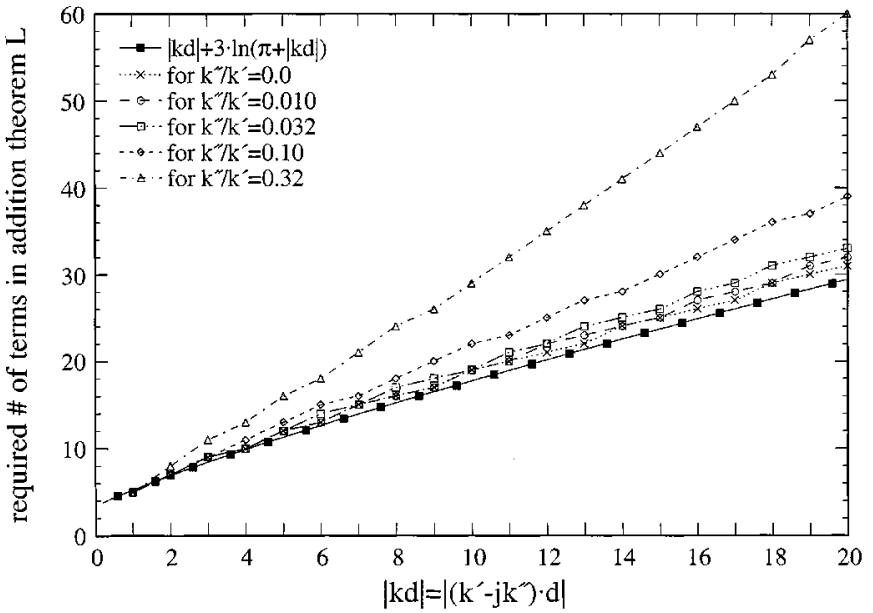

Fig. 2. Number of terms needed in (4) as a function of $|k d|$ for a relative error smaller than $10^{-3}$, depending on the ratio $k^{\prime \prime} / k^{\prime}$ for a complex wavenumber $k=k^{\prime}-j k^{\prime \prime}$. The empirical formula $L=k d+\gamma \ln (\pi+k d)$ is also plotted, with $k d$ replaced by $|k d|$, is also plotted for comparison. Here only the direct interaction (i.e., free-space, but lossy background) is considered.

wavenumber $k_{i}$ and complex source points, although the convergence is slower. This is especially true for the complex distance vectors. While complex wavenumbers $k=k_{i}$ can be handled by slightly increasing the number $L$ of terms in (4) (at least for moderate losses, see Fig. 2), we have not yet found a solution to avoid the generally large upper limit $L$ needed for general complex source points. To further illustrate this, we consider the addition theorem [13], [14], [32]

$$
\begin{aligned}
\frac{e^{-j k R}}{R} & =\frac{e^{-j k \sqrt{(\boldsymbol{X}+\boldsymbol{d}) \cdot(\boldsymbol{X}+\boldsymbol{d})}}}{\sqrt{(\boldsymbol{X}+\boldsymbol{d}) \cdot(\boldsymbol{X}+\boldsymbol{d})}} \\
& =-j k \sum_{l=0}^{L=\infty}(-1)^{l}(2 l+1) j_{l}(k d) h_{l}^{(2)}(k X) P_{l}(\hat{\boldsymbol{d}} \cdot \hat{\boldsymbol{X}})
\end{aligned}
$$

on which the plane wave expansion (4) is based. Theoretically, the expansion in (4) converges as long as $|d|<|X|$ is satisfied, wherein the distance $R$, wavenumber $k, X=\sqrt{X \cdot X}$, and $d=\sqrt{\boldsymbol{d} \cdot \boldsymbol{d}}$ can be arbitrarily complex [32]. As discussed in [13], for real arguments (i.e., real wavenumber $k$, real source point $\boldsymbol{x}^{\prime}$, real group center $\boldsymbol{x}_{m^{\prime}}$, real vectors $\boldsymbol{d}$ and $\boldsymbol{X}$ ), the spherical Bessel function $j_{l}(k d)$ and spherical Hankel function of second kind $h_{l}^{(2)}(k X)$ are approximately of constant magnitude for $l<k d$ and $l<k X$, respectively. The $l$ th-order Legendre polynomial is strictly bounded according to $\left|P_{l}(\hat{\boldsymbol{d}} \cdot \hat{\boldsymbol{X}})\right| \leq 1$. For $l>k d$ and $l>k X$, respectively, $j_{l}(k d)$ decays quickly and $h_{l}^{(2)}(k X)$ grows quickly. Consequently, $L$ must be chosen large enough such that convergence is achieved, but it must not greatly exceed $k X$, or the sum becomes numerically unstable. For the free-space FMM with real arguments (e.g., $k=k_{0}$ ) it has been shown in [13], [14], [17] that, assuming a maximum cluster diameter $d$, approximately $L=k d+\gamma \ln (\pi+k d)$ terms in the series expansion are sufficient; with $\gamma \approx 1$ for a relative error less than $10^{-1}=10 \%, \gamma \approx 3$ for $10^{-3}$ and $\gamma \approx 5$ for $10^{-6}$. If two clusters are situated such that $k X<L$, the clusters are too close for the representation in (4a) and (4b) to be valid [13] and therefore the interactions between those clusters 


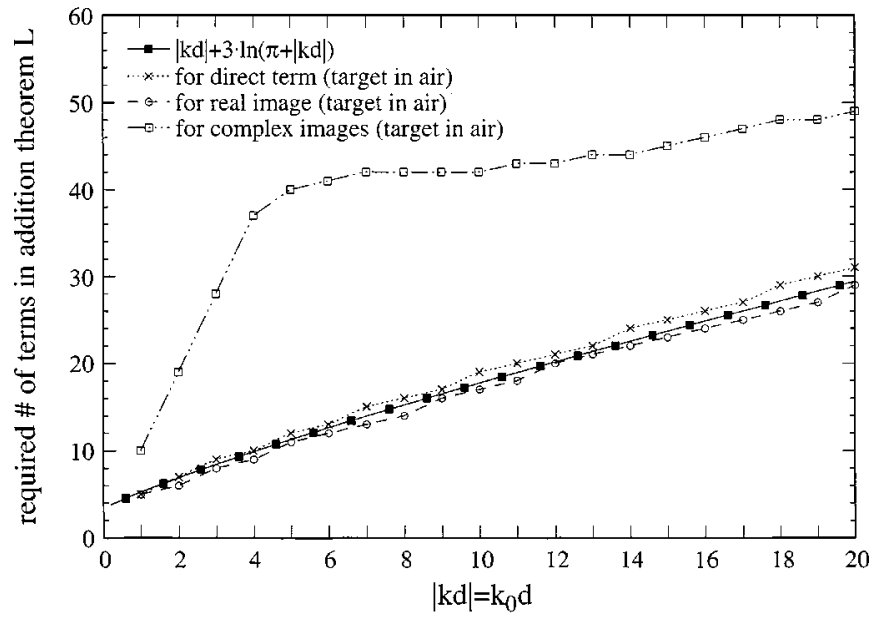

Fig. 3. Number of terms needed in (4) as a function of $|k d|=k_{0} d$ for a relative error smaller than $10^{-3}$ for a scatterer above soil with relative permittivity $\epsilon_{r}=6-j 1$. The source and observation group centers are both located at $z_{m^{\prime}}=z_{m}=0.4 d$ and the separation was set to $k_{0} X_{m^{\prime} m}=L$. In addition to the number of terms required for the direct interaction, the results are given for a real image when considering all complex images.

have to be treated in the sparse part $\left[\mathbf{Z}^{\text {near }}\right]$ of the original MoM impedance matrix (see Section II-A).

For image sources in complex space at least one of the vectors $X$ or $\boldsymbol{d}$ is complex (depending on the specific choice of the image group center $\left.\boldsymbol{x}_{m^{\prime}}\right)$ and the condition $L=k d+\gamma \ln (\pi+k d)$ is no longer valid, which is also the case for real image sources but a lossy background [i.e., complex wavenumber $\left.k=k^{\prime}-j k^{\prime \prime}\right)$ ]. Fig. 2 illustrates the increase in $L$ for a complex wavenumber, depending on the relative losses of the surrounding medium (represented by the ratio $k^{\prime \prime} / k^{\prime}$ ), where here we truncate the series expansion for a relative error less than $10^{-3}$, and the distance between the group centers is set to $|k X|=L$. For moderate losses, the required number of terms in the addition theorem only increases slightly. In contrast, the (in general) significant increase of $L$ for complex images is illustrated in Fig. 3, where we plot the required number of terms $L$ for a target above soil of relative permittivity $\epsilon_{r}=6-j 1$. The source and observation group centers are both located at $z_{m^{\prime}}=z_{m}=0.4 d$, separated by a distance which satisfies $\left|k_{1} X_{m^{\prime} m}\right|=k_{0} X_{m^{\prime} m}=L$. Remembering that $K=2 L^{2}$ terms are utilized in the numerical evaluation of (4a), the computational and memory requirements increase dramatically when using complex images.

Therefore, we searched for an alternative (though approximate) formulation. Considering the fact that the dyadic halfspace Green's function is rigorously accounted for in the "near" interaction matrix $\left[\mathbf{Z}^{\text {near }}\right]$, using a far-field reflection coefficient approximation [30], [31] to include "far" interface interactions seems appropriate (as demonstrated in Section III). Under this assumption it is sufficient to utilize a single real image to approximately account for the reflection at the interface [30], [31]. There is only one set of real image clusters, as opposed to dyadic-component-dependent multiple complex-image locations. This property reduces the overall CPU and memory requirement. In fact, the storage and CPU are approximately twice that of a free-space FMM (see Section III), due to the extra set of image clusters. The location of the (approximate) real image at $\left(x^{\prime}, y^{\prime},-z^{\prime}\right)$ (assuming the interface at $z=0$ ) and the corresponding real image source group are easily determined (Fig. 1). Generalizing the free-space FMM algorithm is now straightforward. In the preprocessing stage [13], [14] we only have to include additional calculations of the translation operators between all image group and observation group centers and the spectral Fourier transforms of the image expansion functions.

To account for the polarization-dependent far-field reflection of a wave traveling from the source point $\boldsymbol{x}^{\prime}$ to the observation point $x$, we introduce the asymptotic expression for the reflection dyadic [30], [31]

$$
\boldsymbol{r}^{v}\left(\boldsymbol{x}, \boldsymbol{x}^{\prime}\right)=\hat{\boldsymbol{h}} \hat{\boldsymbol{h}} R_{T E}^{v}(\vartheta)+[\boldsymbol{I}-\hat{\boldsymbol{h}} \hat{h}] R_{T M}^{v}(\vartheta)
$$

with

$$
\hat{\boldsymbol{h}}=\frac{\hat{z} \times\left(\boldsymbol{x}-\boldsymbol{x}^{\prime}\right)}{\left|\hat{z} \times\left(\boldsymbol{x}-\boldsymbol{x}^{\prime}\right)\right|}
$$

where $\vartheta$ represents the angle between the ray-optical reflection path and the unit vector $\hat{z}$ normal to the interface (Fig. 1). The material properties and the angle $\vartheta$ determine the Fresnel reflection coefficients $R_{T E, T M}^{v}=R_{T E, T M}^{12}$ for a target situated in layer i=1 (e.g., air) or $R_{T E, T M}^{v}=R_{T E, T M}^{21}$ for a target in layer $i=2$ (e.g., soil), respectively. The polarization is denoted as $\mathrm{TE}_{\mathrm{z}}$ (electric field perpendicular to the plane of incidence, i.e., parallel to the unit vector $\hat{\boldsymbol{h}}$ ) and $\mathrm{TM}_{\mathrm{z}}$ (magnetic field perpendicular to the plane of incidence), respectively.

\section{RESULTS}

In all examples below, the scattered fields are computed via the FMM algorithm developed here, as well as with a rigorous MoM solution [7], wherein the dyadic Green's function is characterized rigorously via the method of complex images [26]-[29]. The accuracy, computational complexity, and memory requirements of the FMM are calibrated through comparison with the MoM algorithm. The FMM model discussed here is easily modified to the free-space case [13], [14]; we therefore consider the first target (trihedral) in free space as well, such that the accuracy of the free-space FMM (vis-a-vis the MoM) can be assessed relative to the accuracy of the half-space FMM. This is particularly important because the free-space FMM makes no approximation to the Green's function beyond those in (4), while the half-space FMM code developed here makes an additional approximation (real images) to the half-space Green's function (for the "far" FMM interactions).

Concerning the FMM, recall that $M \propto \sqrt{N}$ clusters are optimal [13], [14], where $N$ is the number of unknowns. For simplicity, we have used $M=2^{q}$ FMM clusters, where $q$ is an integer. While this approach is slightly suboptimal for the two-level FMM applied here, a similar clustering is employed in the multilevel FMM [15]-[17]. As mentioned in Section II-B, the number of terms $L$ in (4b) is determined adaptively, where, for the problems considered here, $L$ is typically in the range 6-15 (when truncating the series for a relative error of $10 \%$ or less, as suggested in [14], [17]). With regard to the MoM, the number of complex-image terms is also determined adaptively, and for the problems considered here 5-15 complex images are 


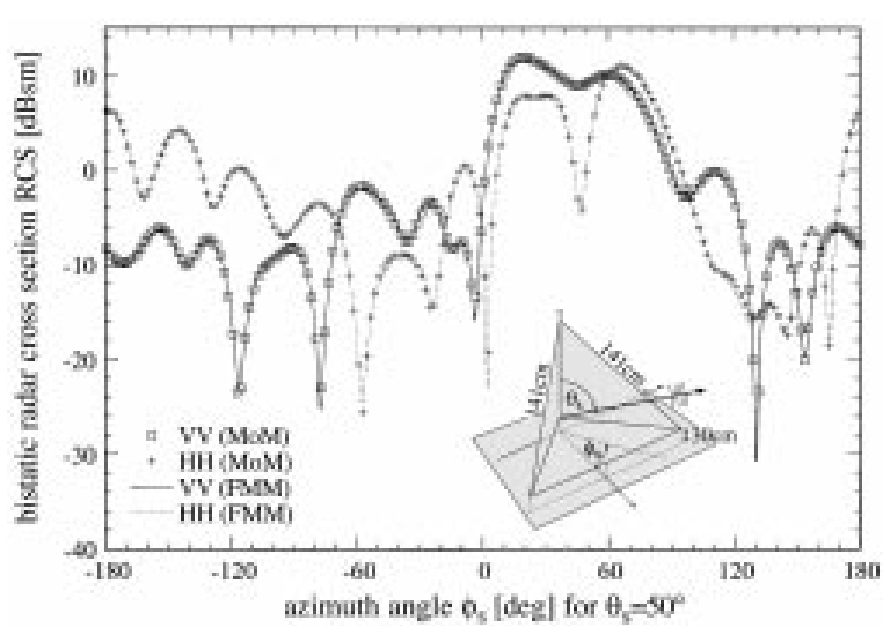

(a)

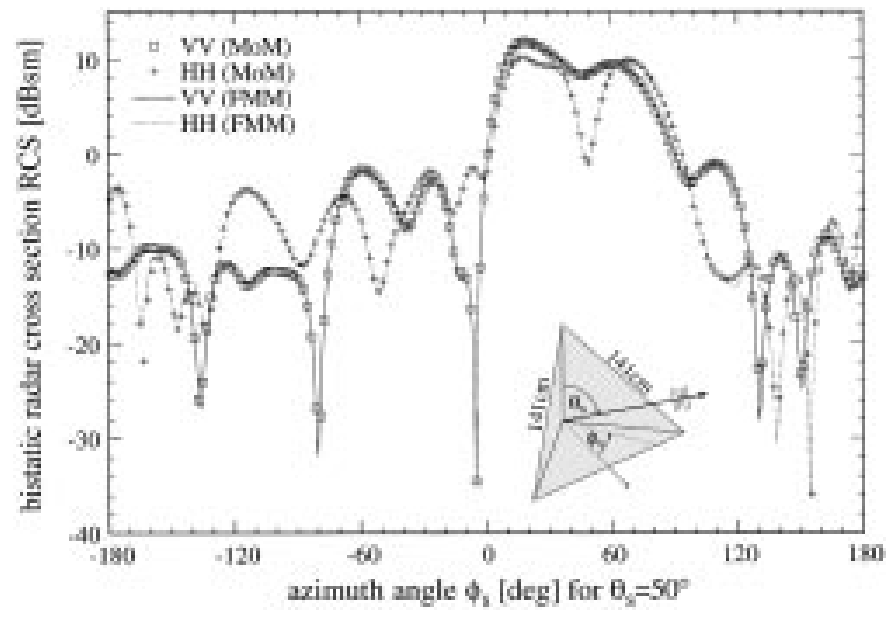

(b)

Fig. 4. Radar cross section of a $90^{\circ}$-trihedral of edge length $100 \mathrm{~cm}$ (hypotenuse $=141 \mathrm{~cm}$ ) with one plate parallel to the $x y$-plane (see inset). The incident plane wave direction is given by $\phi_{i}=20^{\circ}$ (relative to trihedral symmetry plane) and $\theta_{i}=60^{\circ}$ ( $30^{\circ}$ from grazing). The figure shows the bistatic RCS (here we only plot VV and $\mathrm{HH}$ ) for $\theta_{s}=50^{\circ}$ and a frequency of $1 \mathrm{GHz}$ (number of unknowns $N=5202$ ). (a) Trihedral $30 \mathrm{~cm}$ above lossy Yuma soil with $10 \%$ water content [20], [22]. (b) Trihedral in free-space.

typical. We reiterate that the "near" FMM terms employ a complex-image-computed Green's function. Finally, in the MoM solution, we have used a direct (LU-decomposition) solver of order $O\left(N^{3}\right)$ complexity, which could be reduced to $P \cdot O\left(N^{2}\right)$ if a conjugate-gradient solver [2], [8] was applied.

In all the computations, we have assumed linearly polarized plane-wave incidence. The scattered fields are computed in the far zone, via an asymptotic approximation to the half-space dyadic Green's function [30], [34].

\section{A. Trihedral Fiducial Target}

We first consider the trihedral target inset in Fig. 4(a), situated above a lossy half space. The soil is characterized as Yuma soil, with $10 \%$ water content [20], [22]. As depicted in Fig. 4(a), the trihedral is composed of three $45^{\circ}-45^{\circ}-90^{\circ}$ triangles, each with a $1.41 \mathrm{~m}$ hypotenuse. The trihedral is placed $30 \mathrm{~cm}$ above the soil, with one of its triangles parallel to the air-ground interface. Such that the code is tested thoroughly, we first present bistatic results for incidence angles $\theta_{i}=60^{\circ}$ and $\phi_{i}=20^{\circ}$, and the scattered fields are observed for $\theta_{s}=50^{\circ}$ and $-180^{\circ} \leq \phi_{s} \leq$ $180^{\circ}$. The results in Fig. 4(a) present the bistatic scattered fields for VV and $\mathrm{HH}$ polarization, for both the FMM and MoM solutions, at a frequency of $1 \mathrm{GHz}$. At this frequency the hypotenuse is $4.7 \lambda_{0}$, where $\lambda_{0}$ is the free-space wavelength. We see that the agreement between the FMM and MoM results is excellent. Similar agreement was found for the cross-polarized ( $\mathrm{VH}$ and HV) fields, but these are not shown here, such that Fig. 4(a) is easy to read.

While the agreement between the MoM and FMM results is quite good, there are slight discrepancies in some places. While the MoM has few approximations, the FMM algorithm has three additional sources of errors. First, considering (4), the integration over the unit sphere in (4a) is approximated via a $K=2 L^{2}$ point quadrature rule [13]. Further, the sum in (4b) must be truncated appropriately, as discussed in Section II-C. These same approximations are present in the free-space FMM algorithm [13], [14]. The half-space problem, as modeled here, introduces a third source of error. In particular, we have approximated the halfspace Green's function (for the "far" FMM terms) via real images, with appropriate polarization-dependent reflection coefficients. To examine more closely the source of errors in the FMM results of Fig. 4(a), we consider the same trihedral, situated in free space. If the agreement between the MoM and FMM algorithms is better for the free-space case than for the half-space results in Fig. 4(a), then we conclude that the half-space Green's function approximation applied here is inadequate.

In Fig. 4(b) we consider the same case as in Fig. 4(a) but the soil half space is absent. We see by comparing Fig. 4(a) and (b) that the free-space and half-space FMM results demonstrate the same level of accuracy, relative to the MoM computations. This yields further confidence in the accuracy of the real-image approximation to the half-space Green's function, which has been examined previously in the context of the MoM [31]. With regard to the FMM results in Fig. 4(a), we also examined the solution when the reflection term was eliminated (i.e., neglecting reflections in "far" interactions), to see if the real image points were actually important. We found that the accuracy of the half-space FMM results deteriorated dramatically if the real image term was ignored, underscoring the importance and accuracy of this term.

The bistatic results in Fig. 4 provide a thorough test of the half-space FMM's accuracy, but the monostatic RCS is of more interest for practical radar systems. In Fig. 5 we consider the MoM- and FMM-computed backscattered RCS from the same trihedral as considered above, for the half-space and free-space cases, respectively. We consider a frequency of $600 \mathrm{MHz}$, with angles of incidence and scatter $\theta=60^{\circ}$ and $-180^{\circ} \leq \phi \leq$ $180^{\circ}$. We see that for these backscatter results, the agreement between the FMM and MoM results is almost perfect, for both the free-space and half-space cases.

In comparing the results in Figs. 4 and 5, we note that there is often a noticeable difference between the signature of the trihedral in free space and above soil. This is an important issue for radar calibration via such fiducial targets, since most previous results for trihedral scattering have been for the free-space case [7], [35], [36]. The importance of the FMM becomes even more evident as the size of the trihedral increases. For example, 


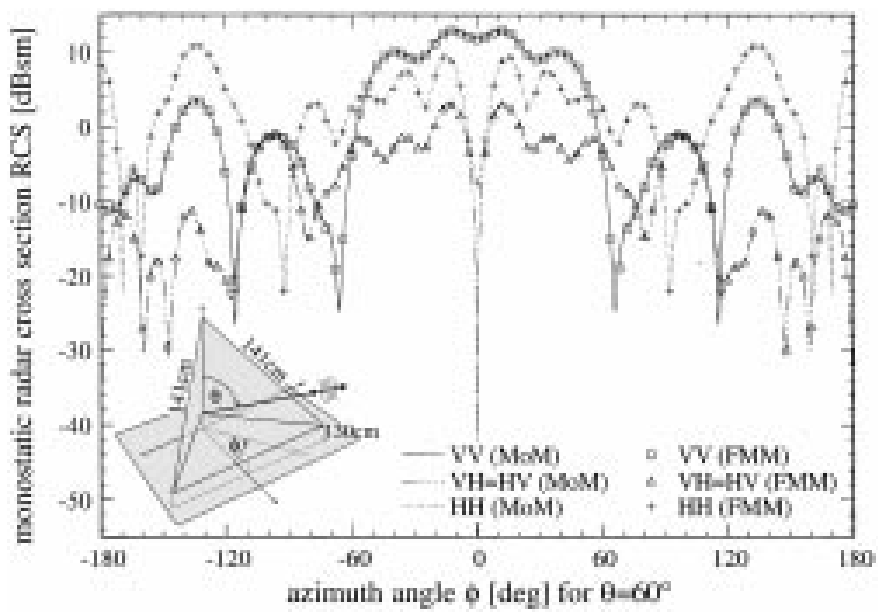

(a)

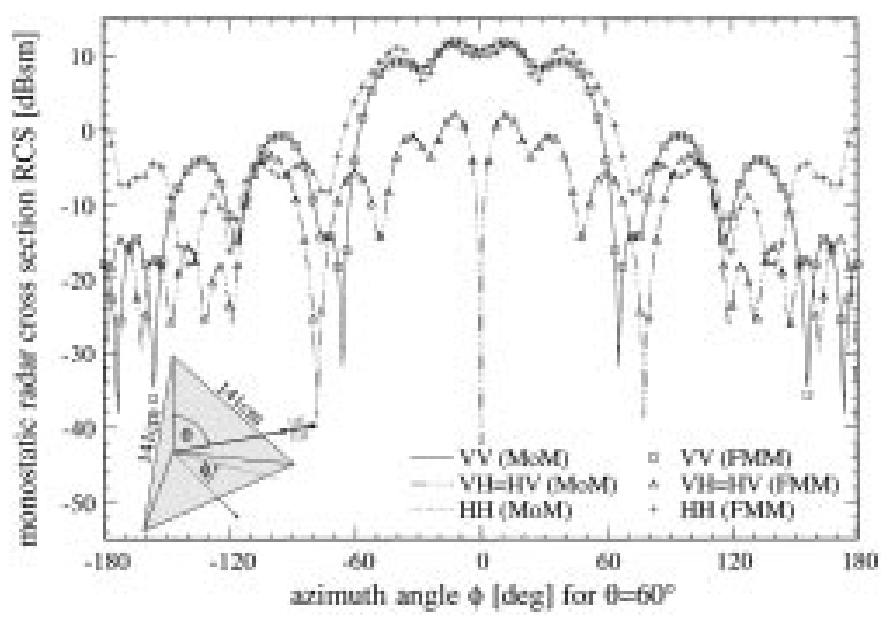

(b)

Fig. 5. Backscattering (monostatic RCS) for the trihedral in Fig. 4 at a frequency of $600 \mathrm{MHz}$ (number of unknowns $N=1770$ ), for varying $\phi=\phi_{i}=\phi_{s}$ and $\theta=\theta_{i}=\theta_{s}=60^{\circ}$. (a) Trihedral $30 \mathrm{~cm}$ above lossy Yuma soil with $10 \%$ water content [20], [22]. (b) Trihedral in free-space.

ultra-wideband synthetic aperture radar (SAR) systems operate over frequencies from 50 to $1200 \mathrm{MHz}$ [20], [22], for which very large trihedrals are required, to account for the lowest frequencies. The efficacy of applying the FMM to this problem has been examined in detail, in a separate paper [37].

Before leaving the trihedral target, we examine the memory requirements and computational speed of the FMM and MoM algorithms, for the results in Fig. 4. In Fig. 6 we compare the FMM and MoM computation speed and memory requirements, for the trihedral in free space as well as over the soil, as a function of the number of unknowns (approximately equal to 1.5 times the number of triangles [3]). The smallest number of unknowns $N=220$ corresponds to a frequency of $200 \mathrm{MHz}$, while the largest $N=9522$ corresponds to a frequency of $1.4 \mathrm{GHz}$. We see that, for the MoM solution, the computation time for the free-space and half-space problems coalesce after $N$ becomes sufficiently large, representing the point at which the $O\left(N^{3}\right)$ LU-decomposition overwhelms the $O\left(N^{2}\right)$ matrix fill time. These results were computed on a HP9000/C240 workstation. The results in Fig. 6 also demonstrate the significant memory savings gained by the FMM, at $N>2500$ for the

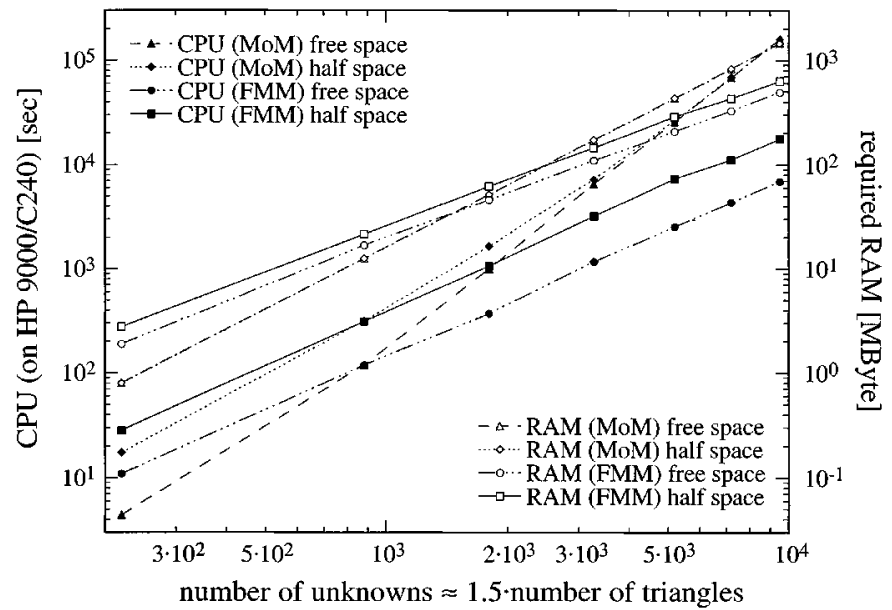

Fig. 6. Required RAM and total CPU time (on HP9000/C240) for bistatic RCS calculations in Fig. 4 (720 scattering angles), for frequencies ranging from 200 to $1400 \mathrm{MHz}$ (corresponding to $N=220-9522$ unknowns) using MoM (with LUD) and FMM. The CPU time for the MoM solution at $f>1000 \mathrm{MHz}$ $(N>5202)$ has been extrapolated.

half-space problem. Finally, we note that the memory requirements and computation time of the half-space FMM, as formulated here, is about twice that of the free-space code. This is attributed to the extra set of image sources, at real spatial positions, which are absent for the free-space problem.

\section{B. Buried 55-Gallon Drum}

We consider a 55-gallon drum (90-cm length, 60-cm diameter) buried with its axis parallel to the air-ground interface. Further, the axis of the target is at a depth of $100 \mathrm{~cm}$. For this example we consider Puerto Rico soil, as reported in [38], with $10 \%$ water content by weight. The wavelength in this soil is reduced in size by more than a factor of two (relative to free space), due to the soil's relatively high dielectric constant between about $\epsilon_{r}^{\prime}=5$ and $\epsilon_{r}^{\prime}=6.5$ [38]. We consider normal incidence relative to the air-ground interface, addressing separately the case of perpendicular $(\mathrm{V})$ and parallel $(\mathrm{H})$ polarization relative to the target axis. In Table I we present the copolarized VV and HH backscattered RCS of the target, as a function of frequency, from 100-1000 MHz. The MoM results are only computed up to $500 \mathrm{MHz}$, above which the MoM computation time (Fig. 7) becomes prohibitive. However, for frequencies below $600 \mathrm{MHz}$, there is excellent agreement between the rigorous MoM solution and the FMM results.

In Fig. 7 we plot the computation time and memory requirements of the MoM and FMM algorithms, for the results in Table I. All MoM computation times for frequencies over 500 $\mathrm{MHz}$ are estimated. These results, which were computed on a SGI Origin 2000 supercomputer (only one processor used), demonstrate the significant gains afforded by the FMM, in both computation time and memory. Upon close inspection of the FMM results in Fig. 7, one notices slight undulations in the data. This, as discussed above, is due to the fact that we utilize $M=2^{q}$ FMM clusters, where $q$ is an integer. As $N$ is varied, $M=2^{q}$ approaches and recedes the optimal $M \propto \sqrt{N}$, causing a slight undulation in the results (as the algorithm, with $M=2^{q}$ clusters, approaches and recedes optimality). 


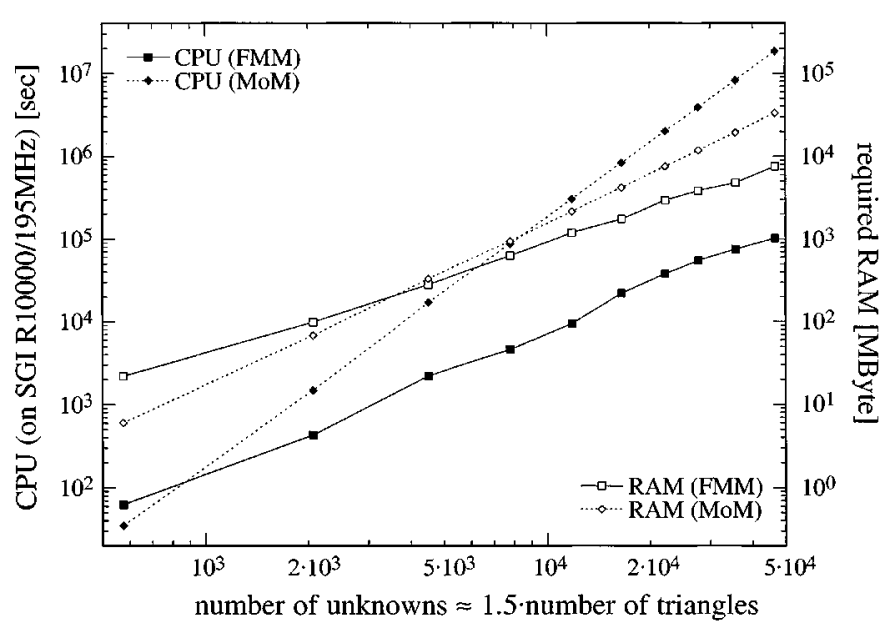

Fig. 7. Required RAM and CPU time (on SGI R10000/195 MHz) for monostatic RCS calculations of 55-gallon drum in Table I using MoM (with LUD) and FMM, i.e., $N=576-46623$ unknowns corresponding to $f=100-1000 \mathrm{MHz}$. The CPU time for the MoM solution at $f>500 \mathrm{MHz}$ $(N>11841)$ has been extrapolated.

TABLE I

BACKSCATTERING (RCS IN [dBsm]) OF A 55-GALLON DRUM (LENGTH $=$ $90 \mathrm{~cm}$, DIAMETER $=60 \mathrm{~cm})$ BURIED IN PUERTO RICO CLAY $(10 \%$ WATER BY WEIGHT) [38] AS A FUNCTION OF FREQUENCY FOR A NORMALlY INCIDENT Plane WaVe $\left(\theta=0^{\circ}\right)$. The Drum Is Situated With ItS Symmetry Axis $100 \mathrm{~cm}$ Below the HalF-SPaCe Boundary, Parallel to THE INTERFACE. THE INCIDENT ELECTRIC FIELD IS PERPENDICULAR TO THE CYLINDER AXIS FOR VV AND PARALLEL FOR HH POLARIZATION

\begin{tabular}{c|c|c|c|c|c}
\hline$f[\mathrm{MHz}]$ & $N$ (unknowns) & VV (MoM) & HH (MOM) & VV (FMM) & HH (FMM) \\
\hline 100 & 576 & -15.916 & -13.600 & -15.917 & -13.598 \\
\hline 200 & 2070 & -13.238 & -12.658 & -13.247 & -12.682 \\
\hline 300 & 4506 & -12.643 & -12.468 & -12.649 & -12.469 \\
\hline 400 & 7812 & -13.276 & -13.156 & -13.321 & -13.098 \\
\hline 500 & 11841 & -14.738 & -14.549 & -14.754 & -14.526 \\
\hline 600 & 16536 & - & - & -16.495 & -16.314 \\
\hline 700 & 22200 & - & - & -18.248 & -18.149 \\
\hline 800 & 27687 & - & - & -20.119 & -20.072 \\
\hline 900 & 35568 & - & - & -22.137 & -22.102 \\
\hline 1000 & 46623 & - & - & -24.181 & -24.127 \\
\hline
\end{tabular}

\section{Buried Model Unexploded Ordnance (UXO)}

The final set of results correspond to an MK 146 MOD 2 U.S. Navy projectile, with dimensions and shape shown in the inset in Fig. 8, buried in Yuma soil with 5\% water content [20], [22], the target symmetry axis in the $y z$-plane. The plane wave is incident at $\phi_{i}=-90^{\circ}$ (in the $y z$-plane) and $\theta_{i}=60^{\circ}$, and the bistatic scattered fields are observed at angles $\theta_{s}=60^{\circ}$ and $-180^{\circ} \leq \phi_{s} \leq 180^{\circ}$, with a $500-\mathrm{MHz}$ operating frequency. The MoM versus FMM computation and memory requirements are similar to those found for the buried drum (Fig. 7) and the trihedral (Fig. 6) and therefore are not shown here, for brevity.

Considering the results in Fig. 8, we see that the FMM and MoM algorithms agree almost exactly. The depth and orientation of the target in Fig. 8 is realistic (given the target size), but this is a very favorable case for the FMM. In particular, recall that the additional approximation we have introduced [beyond the usual FMM approximations in (4)] is the use of a single real image to represent the reflection at the air-ground interface. This term is needed to account for multiple interactions of

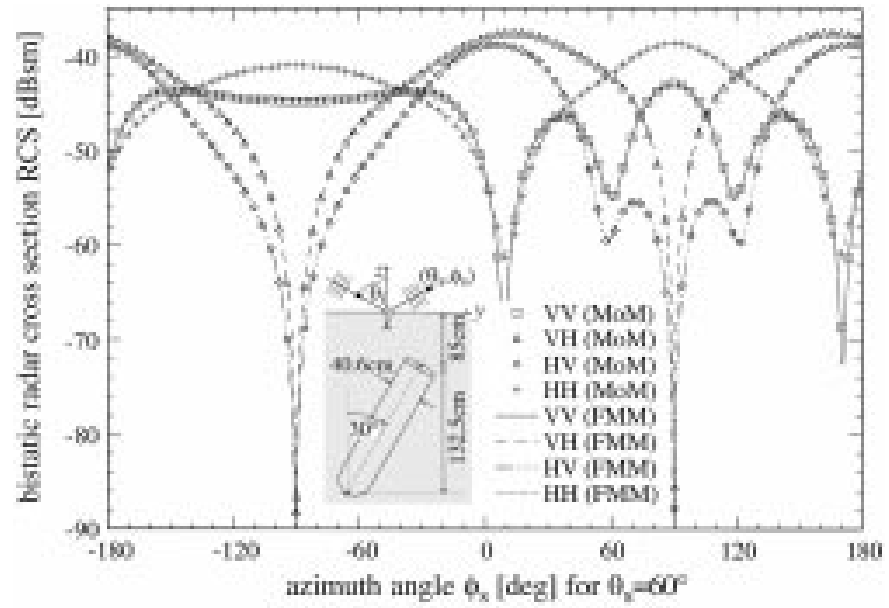

Fig. 8. Bistatic radar cross section of a model UXO (cylinder with hemispherical endcap) of length $153 \mathrm{~cm}$ and diameter $40.6 \mathrm{~cm}$ (see inset). The model UXO is buried in Yuma soil of 5\% water content [20], [22] with the target symmetry axis in the $y z$-plane, at an angle of $30^{\circ}$ relative to the $z$-axis and the nose down at a depth of $z=-217.5 \mathrm{~cm}$. The RCS is plotted for varying $\phi_{s}$ at an angle $\theta_{s}=60^{\circ}$ (30 from grazing) and a frequency of $500 \mathrm{MHz}$ (corresponding to fwr $N=7167$ ), assuming a plane wave incident at $\phi_{i}=-90^{\circ}$ (in $y z$-plane) and $\theta_{i}=60^{\circ}$.

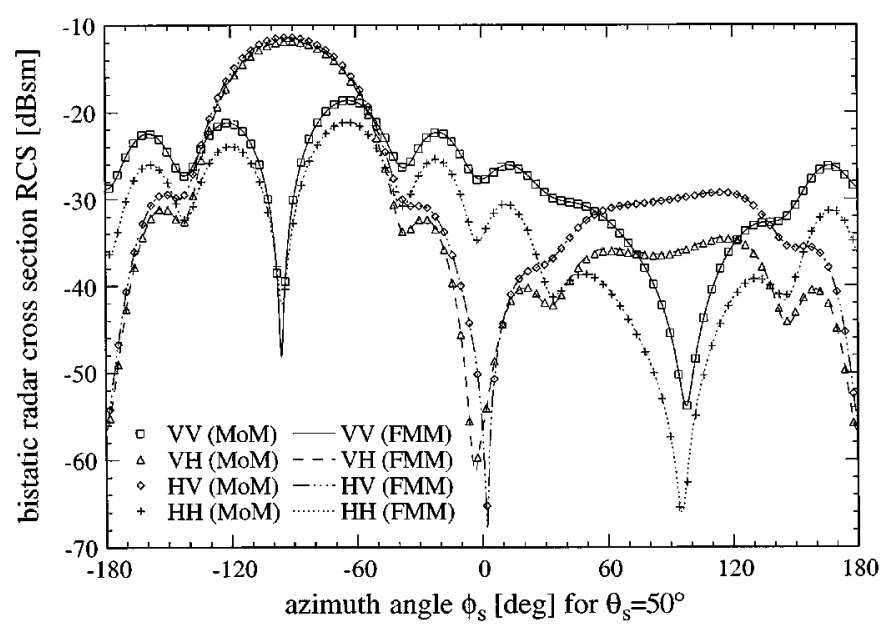

Fig. 9. Same as Fig. 8 but using an angle of $75^{\circ}$ between target axis and $z$-direction $\left(15^{\circ}\right.$ tilt relative to horizontal $x y$-plane), the nose down at a depth of $z=-89.3 \mathrm{~cm}$ (the closest distance between target and interface is $30 \mathrm{~cm}$ ), and a plane wave incident from $\phi_{i}=0^{\circ}$ (in $x z$-plane) and $\theta_{i}=60^{\circ}$ (compare to inset in Fig. 8). The bistatic RCS is plotted for varying $\phi_{s}$ at an angle $\theta_{s}=50^{\circ}$ (40 from grazing).

electromagnetic energy between the target and air-ground interface. For the lossy soil, and relatively deep target, such interactions are less important. Consequently, as a better test of the half-space FMM developed here, we consider the same ordnance considered in Fig. 8, with the angle between the target axis and z-axis (see Fig. 8) set to $75^{\circ}$. Moreover, the closest distance between the target and the interface is $30 \mathrm{~cm}$, rather than $75 \mathrm{~cm}$ in Fig. 8. While such a shallow depth is unlikely for a target of this size, the target-interface interactions are considerably more important for this case [although not as important as for a target above the interface, as in Figs. 4(a) and 5(a)]. Results are shown in Fig. 9, where we consider the same frequency, but modified incidence $\left(\theta_{i}=60^{\circ}\right.$ and $\phi_{i}=0^{\circ}$, i.e., in the $x z$-plane $)$ and scattering angles $\left(\theta_{s}=50^{\circ}\right)$. We note that the bistatic RCS of this shallow target is considerably larger than that of the deeper 
target (Fig. 8), and the agreement between the MoM and FMM results is again excellent.

\section{CONCLUSION}

The FMM has been extended to the case of 3-D perfectly conducting targets situated above or within a lossy half space. The "near" FMM terms are handled as in a MoM analysis, wherein the half-space dyadic Green's function is characterized rigorously via the method of complex images [26]-[29]. For the "far" FMM terms, the reflected terms in the dyadic half-space Green's function are approximated via real images with polarization-dependent amplitudes [30]; this technique having proven useful in previous MoM research [31]. The location of the real image is the same for each component of the dyadic. Thus, in addition to being accurate, this approximation yields significantly reduced computational and memory requirements.

Results were presented for targets above and below a half space, with good agreement vis-a-vis a reference MoM solution. Nevertheless, the subsurface problem is worthy of further examination. In particular, the real images are required for accurate treatment of the target-interface interaction. However, for deep targets and for relatively lossy soils, the images may be unnecessary, yielding a half-space FMM that replicates the free-space version [13], [14] (although the incident field and the scattered far-field calculations are slightly more complicated). This is likely to be a strong function of target type, depth, and orientation, as well as of the detailed soil characteristics. Moreover, recall that the "far" FMM interactions are handled via the spectral propagator in (4b). If the target is buried in a lossy half space (i.e., complex $k_{i}$ ), $T_{L}$ is small after sufficiently large intercluster distance $X_{m^{\prime} m}$. Consequently, for the buried-target case, a further algorithm acceleration and reduction in memory can be achieved by ignoring intercluster interactions for sufficiently large $X_{m^{\prime} m}$.

\section{REFERENCES}

[1] R. F. Harrington, Field Computations by Moment Methods. New York: Macmillan, 1968.

[2] A. F. Peterson, S. L. Ray, and R. Mittra, Computational Methods for Electromagnetics. Piscataway, NJ: IEEE Press, 1998.

[3] S. M. Rao, D. R. Wilton, and A. W. Glisson, "Electromagnetic scattering from surfaces of arbitrary shape," IEEE Trans. Antennas Propagat., vol. 30, pp. 409-418, May 1982.

[4] D. R. Wilton, S. M. Rao, A. W. Glisson, D. H. Schaubert, O. M. Al-Bundak, and C. M. Butler, "Potential integrals for uniform and linear source distributions on polygonal and polyhedral domains," IEEE Trans. Antennas Propagat., vol. 32, pp. 276-281, Mar. 1984.

[5] K. A. Michalski and D. Zheng, "Analysis of microstrip resonators of arbitrary shape," IEEE Trans. Microwave Theory Tech., vol. 40, pp. 112-119, Jan. 1992.

[6] - "Electromagnetic scattering and radiation by surfaces of arbitrary shape in layered media, Parts I and II," IEEE Trans. Antennas Propagat., vol. 38, pp. 335-352, Mar. 1990.

[7] N. Geng, M. Ressler, and L. Carin, "Wideband VHF scattering from a trihedral reflector situated above a lossy dispersive half space," IEEE Trans. Geosci. Remote Sensing, vol. 37, pp. 2609-2617, Sept. 1999.

[8] T. K. Sarkar and E. Arvas, "On a class of finite step iterative methods (conjugate directions) for the solution of an operator equation arising in electromagnetics," IEEE Trans. Antennas Propagat., vol. 33, pp. 1058-1066, Oct. 1985.

[9] F. X. Canning, "Improved impedance matrix localization method," IEEE Trans. Antennas Propagat., vol. 41, pp. 659-667, May 1993.

[10] R. L. Wagner and W. C. Chew, "A study of wavelets for the solution of electromagnetic integral equations," IEEE Trans. Antennas Propagat., vol. 43, pp. 802-810, Aug. 1995.
[11] A. Boag and R. Mittra, "Complex multipole beam approach to electromagnetic scattering problems," IEEE Trans. Antennas Propagat., vol. 42, pp. 366-372, Mar. 1994.

[12] E. Bleszynski, M. Bleszynski, and T. Jaroszewicz, "AIM: Adaptive integral method compression algorithm for solving large scale electromagnetic scattering and radiation problems," Radio Sci., vol. 31, pp. 1225-1251, Sept.-Oct. 1996.

[13] R. Coifman, V. Rokhlin, and S. Wandzura, "The fast multipole method for the wave equation: A pedestrian prescription," IEEE Antennas Propagat. Mag., vol. 35, pp. 7-12, June 1993.

[14] J. M. Song and W. C. Chew, "Fast multipole method solution using parametric geometry," Micr. Opt. Techn. Lett., vol. 7, pp. 760-765, Nov. 1994.

[15] — - "Multilevel fast multipole algorithm for solving combined field integral equations of electromagnetic scattering," Micr. Opt. Techn. Lett., vol. 10, pp. 14-19, Sept. 1995.

[16] J. M. Song, C. C. Lu, W. C. Chew, and S. W. Lee, "Fast Illinois solver code (FISC)," IEEE Antennas Propagat. Mag., vol. 40, pp. 27-33, June 1998.

[17] J. M. Song, C. C. Lu, and W. C. Chew, "Multilevel fast multipole algorithm for electromagnetic scattering by large complex objects," IEEE Trans. Antennas Propagat., vol. 45, pp. 1488-1493, Oct. 1997.

[18] L. Gurel and M. I. Aksun, "Electromagnetic scattering solution of conducting strips in layered media using the fast multipole method," IEEE Microwave Guided Wave Lett., vol. 6, pp. 277-279, Aug. 1996.

[19] J. S. Zhao, W. C. Chew, C. C. Lu, E. Michielssen, and J. Song, "Thinstratified medium fast-multipole algorithm for solving microstrip structures," IEEE Trans. Microwave Theory Tech., vol. 46, pp. 395-403, Apr. 1998.

[20] L. Carin, N. Geng, M. McClure, J. Sinchina, and L. Nguyen, "Ultrawideband synthetic aperture radar for mine field detection," IEEE Antennas Propagat. Mag., vol. 41, pp. 18-33, Feb. 1999.

[21] C. C. Chen and L. Peters, "Buried unexploded ordnance identification via complex natural resonances," IEEE Trans. Antennas Propagat., vol. 45, pp. 1645-1654, Nov. 1997.

[22] S. Vitebskiy, L. Carin, M. A. Ressler, and F. H. Le, "Ultra-wideband, short-pulse ground-penetrating radar: Simulation and measurement," IEEE Trans. Geosci. Remote Sensing, vol. 35, pp. 762-772, May 1997.

[23] S. Vitebskiy, K. Sturgess, and L. Carin, "Short-pulse plane-wave scattering from buried perfectly conducting bodies of revolution," IEEE Trans. Antennas Propagat., vol. 44, pp. 143-151, Feb. 1996.

[24] N. Geng and L. Carin, "Wideband electromagnetic scattering from a dielectric BOR buried in a layered lossy, dispersive medium," IEEE Trans. Antennas Propagat., vol. 47, pp. 610-619, Apr. 1999.

[25] Y. Rahmat-Samii, R. Mittra, and P. Parhami, "Evaluation of Sommerfeld integrals for lossy half-space problems," Electromagn., vol. 1, no. 1, pp. $1-28,1981$

[26] Y. L. Chow, J. J. Yang, D. G. Fang, and G. E. Howard, "A closed-form spatial Green's function for the thick microstrip substrate," IEEE Trans. Microwave Theory Tech., vol. 39, pp. 588-592, Mar. 1991.

[27] J. J. Yang, Y. L. Chow, and D. G. Fang, "Discrete complex images of a three-dimensional dipole above and within a lossy ground," Proc. Inst. Elect. Eng.-H, vol. 138, pp. 319-326, Aug. 1991.

[28] R. M. Shubair and Y. L. Chow, "A simple and accurate complex image interpretation of vertical antennas present in contiguous dielectric halfspaces," IEEE Trans. Antennas Propagat., vol. 41, pp. 806-812, June 1993.

[29] M. I. Aksun, "A robust approach for the derivation of closed-form Green's functions," IEEE Trans. Microwave Theory Tech., vol. 44, pp. 651-658, May 1996.

[30] I. V. Lindell, Methods for Electromagnetic Field Analysis. Piscataway, NJ: IEEE Press, 1995.

[31] U. Jakobus, Erweiterte Momentenmethode zur Behandlung kompliziert aufgebauter und elektrisch groer elektromagnetischer Streuprobleme (in in German), VDI Forschungsberichte, 1994.

[32] M. Abramowitz and I. A. Stegun, Handbook of Mathematical Functions. New York: Dover, 1970.

[33] A. D. McLaren, "Optimal numerical integration on a sphere," Math. Computation, vol. 17, pp. 361-383, 1963.

[34] L. B. Felsen and N. Marcuvitz, Radiation and Scattering of Waves. Englewood Cliffs, NJ: Prentice-Hall, 1973.

[35] K. Sarabandi and C. C. Chiu, "Optimum corner reflectors for calibration of imaging radars," IEEE Trans. Antennas Propagat., vol. 44, pp. 1348-1361, Oct. 1996.

[36] T. Griesser and C. A. Balanis, "Backscatter analysis of dihedral corner reflectors using physical optics and the physical theory of diffraction," IEEE Trans. Antennas Propagat., vol. AP-35, pp. 1137-1147, Oct. 1987. 
[37] N. Geng, A. Sullivan, and L. Carin, "Fast multipole method for scattering from 3D PEC targets situated in a half-space environment," Micr. Opt. Techn. Lett., vol. 21, pp. 399-405, Feb. 1999.

[38] J. E. Hipp, "Soil electromagnetic parameters as functions of frequency, soil density, and soil moisture," Proc. IEEE, vol. 62, pp. 98-103, Jan. 1974.

Norbert Geng (S'91-M'96) was born May 14, 1965 in Lauchringen, Germany. He received the Dipl.-Ing. and Dr.-Ing. degrees in electrical engineering from the University of Karlsruhe, Germany, in 1991 and 1996, respectively.

From 1991 to 1996 he was with the Institute for Microwaves and Electronics at the University of Karlsruhe, working on full-wave propagation modeling for radio communications systems. In January 1997 he joined the Department of Electrical and Computer Engineering at Duke University, Durham, NC, as a visiting post-doctoral Fellow for 18 months. While visiting Duke University, and for another 18 months back at the University of Karlsruhe, his research focused on numerical techniques in computational electromagnetics for planarstratified media. In January 2000 he joined SIEMENS Corporate Technology in Munich, Germany, working as a Research Scientist on the design of broadband wireless communications systems.

Dr. Geng received the Mannesmann Innovation Award in 1997 for his Ph.D. dissertation.
Anders Sullivan (M'93) was born August 9, 1963, in Staten Island, NY. He received the B.S. and M.S. degrees in aerospace engineering from the Georgia Institute of Technology, Atlanta, GA, in 1985 and 1987, respectively, and the Ph.D. degree in electromagnetics from Polytechnic University, Brooklyn, NY, in 1997.

From 1988 through May 1998, he was with the Air Force Research Laboratory at Eglin Air Force Base, FL. From June 1998 through September 1999 he was with the Electrical Engineering Department at Duke University as a Research Associate. Since September 1999, he has been with the Army Research Laboratory in Adelphi, MD. His current research interests include modeling complex targets and short-pulse scattering.

Dr. Sullivan is a member of the Tau Beta Pi and Sigma Gamma Tau.

Lawrence Carin (F'01) was born March 25, 1963, in Washington, DC. He received the B.S., M.S., and Ph.D. degrees in electrical engineering from the University of Maryland, College Park, in 1985, 1986, and 1989, respectively.

In 1989 he joined the Electrical Engineering Department at Polytechnic University (Brooklyn) as an Assistant Professor and became an Associate Professor there in 1994. In September 1995 he joined the Electrical Engineering Department at Duke University, where he is an Associate Professor. His current research interests include short-pulse scattering, subsurface sensing, and wave-based signal processing.

Dr. Carin is currently an Associate Editor of the IEEE TRANSACTIONS ON Antennas and Propagation. He is a member of the Tau Beta Pi and Etea Kappa $\mathrm{Nu}$. 